\title{
Not all through-the-scope endoscopic clipping devices are born equal: some can be rotated while others cannot
}

\author{
Shou-jiang Tang
}

Received: 20 March 2013/Accepted: 8 April 2013/Published online: 30 April 2013

(c) Springer Science+Business Media New York 2013

To the Editor,

We appreciate Drs. Saxena and Khashab's [1] comments on our benchtop comparison study data of three commonly used through-the-scope (TTS) endoclips [2]. QuickClip2 long (Olympus America, Center Valley, PA, USA) and Instinct clip (Cook Medical, Winston-Salem, NC, USA) are designed and built to provide precise rotational capability. On the other hand, the Resolution clip (Boston Scientific, Natick, MA, USA) is not built to rotate. The lack of ability to rotate had been observed since its release through clinical observation. Our study objectively demonstrated and confirmed this long-held practical observation. In our study, we tested the Resolution clip for rotational ability with and without a sheath.

Based on their experience, Drs. Saxena and Khashab indicated that the Resolution clip can be rotated with careful technique. Their tricks include removing the outer plastic sheath from the clip prior to use, having the endoscopist rotate the metal cable of the clip close to the biopsy valve, providing small incremental turns, and expecting a short delay. They correctly pointed out that in our study the clips were rotated at the device handle instead of the metal cable. We elected to rotate the device handle since this is the controlling part of nearly all endoscopic devices. It is intuitive to have the assistant control the clip opening, rotation, and deployment by manipulating the handle. In the past, I tried using a hemostat forceps to turn the metal cable of the Resolution clip and straightened the endoscope. My experience has been mostly frustrating. When the endoscope is looped or when using the duodenoscope, the Resolution clip can barely move, not to mention "dance" smoothly with any maneuver. Many endoscopic devices can be "rotated or jiggered" by rotating the metal cable close to the biopsy valve, yet they are not rotational devices and precisely controlled rotation cannot be achieved. We can drive and turn an automobile by handling the steering wheel, yet we can also move the vehicle by pushing it.

When we performed the study, we video recorded clip movement and hand movement for analysis. Each clip was given equal time to respond and handle rotation was performed by the only one investigator, myself. We tried our best to control possible study bias.

Disclosure Shou-jiang Tang has no conflicts of interest or financial ties to disclose

\section{References}

1. Saxena P, Khashab MA (2013) Can all through-the-scope endoscopic clipping devices be rotated? Yes, they can. Surg Endosc. doi:10.1007/s00464-013-2971-y

2. Daram SR, Tang SJ, Wu R, To SD (2013) Benchtop testing and comparisons among three types of through-the-scope endoscopic clipping devices. Surg Endosc 27(5):1521-1529. doi:10.1007/ s00464-012-2679-4
S. Tang $(\bowtie)$

Division of Gastroenterology, Department of Internal Medicine,

University of Mississippi Medical Center, 2500 North State

Street, Jackson, MS 39216, USA

e-mail: stang@umc.edu 